\title{
Invasive Candidiasis in the Elderly: Considerations for Drug Therapy
}

\author{
B. G. J. Dekkers ${ }^{1} \cdot$ A. Veringa ${ }^{1}$ - D. J. E. Marriott ${ }^{2}$ - J. M. Boonstra ${ }^{1}$ - K. C. M. van der Elst ${ }^{3}$ - F. F. Doukas ${ }^{4,5,6}$. \\ A. J. McLachlan ${ }^{4}$. Jan-Willem C. Alffenaar ${ }^{1}$
}

Published online: 26 July 2018

(c) The Author(s) 2018

\begin{abstract}
Candida infections in the elderly are an important and expanding clinical problem, with significantly higher mortality in this group than in younger patients. The increasing problem of invasive Candida infections may be related to higher prevalence of immunocompromised older people and the emergence of treatment resistance. Older people, especially the frail and critically ill, are at higher risk of medication-related harmful effects due to changes in pharmacokinetics and pharmacodynamics, which may be further complicated by organ dysfunction, diminished homeostatic control, co-morbidities and polypharmacy. Here, we review the available options for the treatment of Candida infections and provide insights into the challenges surrounding the optimal use of antifungal drugs in the elderly.
\end{abstract}

\section{Key Points}

Candida infections are a growing problem in elderly patients, with significant mortality in this group.

Treatment in the elderly requires careful consideration of benefit and harm in the selection of both the drug and dose regimen.

Therapeutic drug monitoring can be recommended for selected antifungal agents.

Jan-Willem C. Alffenaar

j.w.c.alffenaar@umcg.nl

1 Department of Clinical Pharmacy and Pharmacology, University Medical Center Groningen, University of Groningen, PO Box 30.001, 9700 RB Groningen, The Netherlands

2 Department of Clinical Microbiology and Infectious Diseases, St Vincent's Hospital, Sydney, NSW, Australia

3 Department of Clinical Pharmacy, ZGT Hospital Group Twente, Hengelo, The Netherlands

4 Sydney Pharmacy School, University of Sydney, Camperdown, NSW, Australia

5 Department of Pharmacy, Concord Repatriation General Hospital, Sydney, NSW, Australia

6 Department of Infectious Diseases and Microbiology, Concord Repatriation General Hospital, Sydney, NSW, Australia

\section{Introduction}

Candida species are part of the normal human microbiota and can be found in the oropharynx, oesophagus, gastrointestinal tract and vagina. Local or invasive infection can occur as a result of a compromised immune system. Predisposing factors such as cancer, the use of immunosuppressants and broad-spectrum antimicrobial agents, and co-morbidities such as diabetes mellitus are all risk factors for an invasive candida infection and are common in older patients [1]. Although systemic therapy is often indicated, mucosal infections and Candida in urine are not considered to be invasive disease. Invasive Candida infections are associated with high morbidity and mortality [2]. The most important Candida species causing invasive disease are C. albicans, C. glabrata, C. krusei, C. parapsilosis and C. tropicalis [1]. Rapid initiation of appropriate antifungal therapy with adequate exposure is needed to achieve optimal treatment outcomes $[2,3]$. The aim of this review is to discuss the epidemiology of Candida infections in the elderly (patients over the age of 65 years) and provide an overview of diagnostics tests to enable rapid diagnosis. The specific drug classes and their pharmacodynamic and pharmacokinetic properties are discussed and considerations in relation to their use in elderly are highlighted. Finally, this review discusses the importance of an antifungal stewardship (AFS) programme to provide optimal patient care in older people with invasive Candida infections. 


\section{Epidemiology of Invasive Candida Infection}

The incidence of candidaemia and invasive Candida infections in the elderly is increasing. In many studies, over half the cases of candidaemia occur in patients over the age of 65 years, and the mortality is significantly higher in the elderly. A recent large prospective multicentre study of 29 Spanish hospitals showed that the infecting Candida species did not differ between younger and older patients, although patients over 75 years of age received inadequate antifungal treatment more often than younger patients [4]. The overall mortality was significantly higher in older patients. A single-centre study of 140 cases of candidaemia produced similar findings [5]. The 30-day mortality in a 5-year retrospective Italian study of 302 patients with candidaemia was significantly higher in older than in younger patients, with a trend towards a greater antifungal resistance in the older group [1]. Comparable findings have been reported in geographically diverse locations, such as China and Brazil $[6,7]$. The risk factors described for candidaemia in the elderly include chronic pulmonary and cardiovascular disease, chronic renal failure, diabetes mellitus and a higher Charlson Comorbidity Index, whilst the 'classic' risk factors, such as haematological malignancies, neutropenia, transplantation and solid tumours are less common in elderly patients [1,7]. Candidaemia in the elderly is an expanding clinical problem with higher mortality in this group, indicating that more timely diagnosis and rapid initiation of appropriate antifungal therapy is urgently required.

\section{Diagnosis of Invasive Candidiasis}

The diagnosis of candidaemia and invasive Candida infection is currently suboptimal and is a contributing factor to the high mortality rate. Although it remains the diagnostic gold standard, only $50-60 \%$ of blood cultures test positive in patients with invasive Candida infections. Conventional identification methods based on blood and agar plate culture typically require $2-4$ days to discriminate among Candida species. Accurate differentiation between species is critical as it guides antifungal therapy. The increasingly widespread use of MALDI-TOF (Matrix Assisted Laser Desorption Ionization-Time of Flight) technology in microbiology laboratories reduces the time to identification of the organism isolated from $24-48 \mathrm{~h}$ to a time of $3 \mathrm{~h}$ or less and there are now protocols for the identification of Candida directly from positive blood culture bottles, without the need to subculture to agar plates [8]. However, this still relies on a positive blood culture as do other new technologies, for example multiplex polymerase chain reaction (PCR) platforms, which can provide results within $1 \mathrm{~h}$ of culture positivity [8].

The limitations of blood cultures in this setting highlight the need for innovative non-culture diagnostic techniques. The 1,3- $\beta$-D-glucan test measures a cell wall constituent of many fungal species and as such is not specific for invasive Candida infection [9]. The pooled sensitivity and specificity in recent meta-analyses was $75-80$ and $80 \%$, respectively, in patients with proven or probable invasive fungal infections, with even lower sensitivity and specificity for patients with intra-abdominal infections without fungi or yeast $[9,10]$. It is also an expensive test, especially if assays are performed in real-time rather than batched. Other non-culture tests, such as multiplex PCR or the T2 Candida panel [8], target the five to six most common Candida species and therefore have an advantage over 1,3- $\beta$-D-glucan by providing species identification. Non-culture diagnostic tests are not definitive, but may identify patients with invasive candidiasis prior to confirmatory cultures and can detect some infections that would be missed by culture. However, they may also provide false-positive results and identify a significant number of patients at a very low risk of invasive candidiasis without disease and thus must be targeted to the patient population at risk for invasive candidiasis and interpreted in the clinical setting.

\section{Antifungal Treatment in the Elderly}

\subsection{Pharmacodynamic and Pharmacokinetic Considerations in the Elderly}

Optimal management of invasive infectious disease requires careful consideration of the impact of aging on pharmacokinetics and pharmacodynamics. Figure 1 provides a summary of the major factors influencing individualised dose selection in older people to achieve optimal health outcomes from antifungal treatment [11-14]. While a person's numerical age is a poor guide to dosing, most patients are considered in the 'elderly' cohort at the age of 65 years and above. Consideration is needed of both intrinsic and extrinsic factors that influence pharmacological response and health outcomes.

\subsubsection{Variability in Pharmacokinetics and Pharmacodynamics in Older People}

Variability in pharmacokinetics in older people is a major contributor in the variable response to antifungal treatment [11]. While pharmacogenetic variation (as observed for some antifungal agents, for example) has been identified as important, it is less clinically significant in older people. 


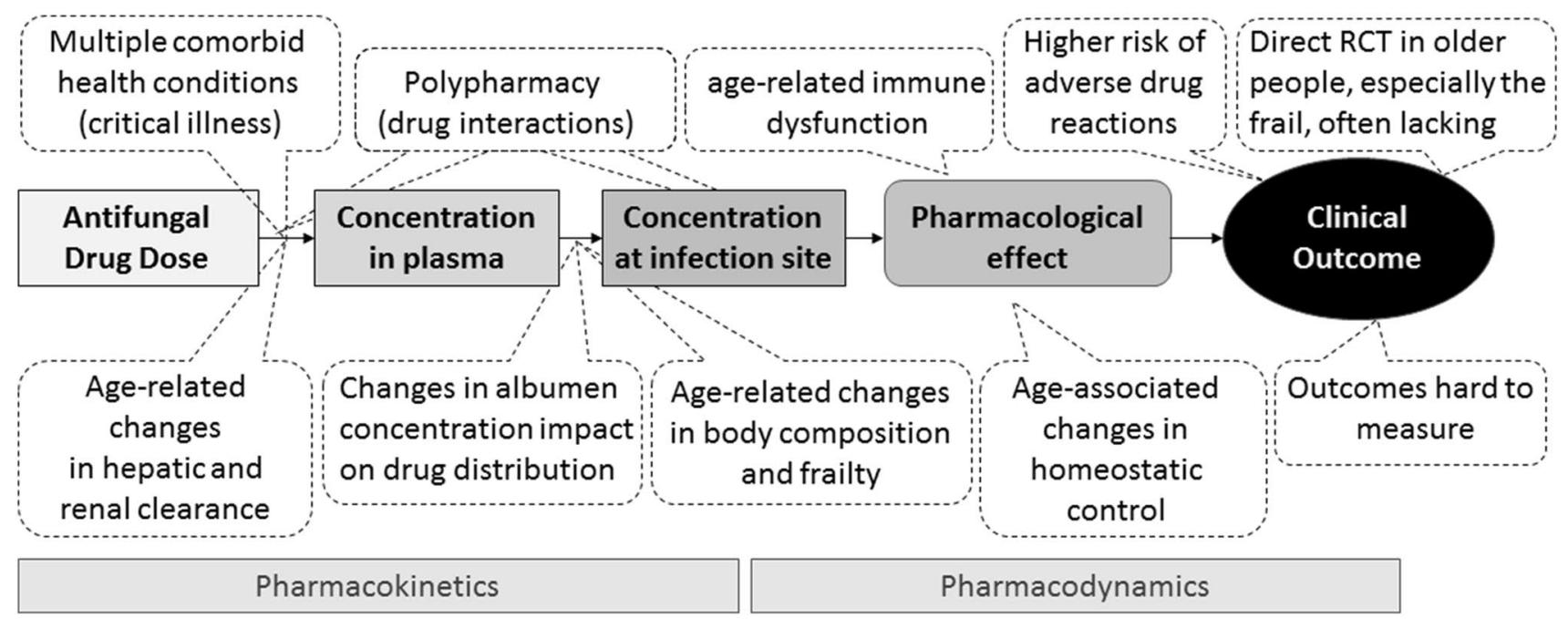

Fig. 1 Pharmacodynamic and pharmacokinetic considerations in older people. $R C T$ randomised controlled trial

Here, extrinsic factors, such as changes in clearance due to the impact of the ageing liver and kidney and drug-drug interactions due to polypharmacy, have a greater impact on the pharmacokinetics of antifungal drugs [11-16]. Frailty has been identified as a major factor contributing to pharmacokinetic variability [13, 17], including for some antimicrobial agents [18]. Age-associated changes in body composition can influence pharmacokinetics and a reduction in serum albumin concentration can have an impact on drug protein binding and therefore on the unbound drug concentration [14]. Hepatic clearance by cytochrome P450 (CYP) is more likely to be impaired in the elderly, although this may be secondary to reduced hepatic blood flow and liver size which occur with advancing age [43]. Measuring unbound drug concentrations is strongly advocated when monitoring antimicrobial drug therapy in older or critically ill patients and for interpreting clearance in older people $[19,20]$.

Pharmacodynamic variability is common in older people as well $[13,14]$. Older people are generally considered more sensitive to the effects of medicines, especially sedatives $[11,14]$. Age-related changes in immune function (so-called immunosenescence) have the potential to impact on response to antimicrobial treatment [14, 21]. The impact on antifungal pharmacodynamics is not well-understood but can contribute to the variability in response.

Older people, especially the frail and critically ill, are at substantially higher risk of medication-related harmful effects from treatment $[19,21]$. Age-related changes in pharmacokinetics and pharmacodynamics are a major contributor, further complicated by organ dysfunction, diminished homeostatic control as well as co-morbidities and polypharmacy $[11,13,14]$.

\subsubsection{Evidence to Guide Dose Individualisation in Older People}

There is limited pharmacokinetic information that is directly relevant to frail older people, with multiple health problems, organ dysfunction and polypharmacy. High-quality trial evidence is lacking, so clinicians have to rely on clinical pharmacological principles to guide drug and dose selection [13]. Stott and Hope [22] highlight the challenge of limited real-word data to guide therapeutic drug monitoring (TDM) and the lack of information linking target antifungal concentrations and clinical outcomes, such as the fungal prophylaxis or treatment of invasive fungal disease. Aging is an important consideration in both drug and dose regimen selection, together with careful monitoring of benefit and harm, especially in frail critically ill older patients (Fig. 1).

\subsection{Drugs}

\subsubsection{Echinocandins}

The echinocandins inhibit the synthesis of $1,3-\beta$-D-glucan. The currently available drugs of this class include caspofungin, anidulafungin and micafungin. These agents target the fungal cell wall, instead of cell components which are also present in mammalian cells, resulting in reduced toxicity [23]. The echinocandins are recommended by the Infectious Diseases Society of America (IDSA) and the European Society for Clinical Microbiology and Infectious Diseases (ESCMID) guidelines as the first-line treatment of invasive candidiasis [2,3]. As a class, these agents are generally well-tolerated. Adverse events most frequently observed in patients are nausea, elevated hepatic enzymes, rash and phlebitis [24]. The favourable characteristics of 
the echinocandins when compared with other antifungal agents include fungicidal activity, limited resistance, excellent safety profile and few drug-drug interactions [25-27]. The echinocandins are administered once daily at fixed doses and are only available for parenteral use [28]. Dose adjustments for the echinocandins are not required for older patients. Studies in healthy elderly demonstrated no significant change in pharmacokinetics compared with healthy non-elderly for all three echinocandins. Caspofungin and anidulafungin are effective and safe for the treatment of invasive candidiasis in elderly patients, despite higher disease severity scores, greater likelihood of decreased organ function and greater likelihood of concomitant drug use [29, 30].

The echinocandins are not cleared renally, and therefore dose adjustment in older patients with renal impairment is not required. Drug-drug interactions are uncommon since all three agents are not transported by P-glycoproteins [28]. Anidulafungin is not hepatically metabolised and caspofungin and micafungin undergo minimal degradation by CYP isoenzymes. Nonetheless, caspofungin degradation has been shown to be enhanced by strong CYP3A4 inducers and both agents may inhibit CYP3A4 to some extent. The limited drug-drug interactions in geriatric patients are desirable considering the frequent use of concomitant drugs. Although the metabolism of caspofungin via CYP is a minor pathway, dose adjustment for caspofungin is recommended in patients with mild or severe liver impairment. The lack of an oral formulation and daily administration makes the echinocandins less suitable for ambulatory use. Comparative trials between the echinocandins, primarily micafungin and fluconazole demonstrated potential superiority of echinocandins over fluconazole in prevention of fungal infections [31]. The use of echinocandins in the ambulatory setting is currently being investigated for a new 'long-acting' echinocandin, rezafungin. Rezafungin has the same favourable characteristics as the other echinocandins but has been developed for once-weekly intravenous or subcutaneous administration [32].

\subsubsection{Azoles}

The azole antifungal agents include fluconazole, itraconazole, voriconazole, posaconazole and isavuconazole. The mechanism of action is the inhibition of lanosterol $14 \alpha$-demethylase, blocking the synthesis of ergosterol and resulting in impaired membrane stability and the accumulation of precursors leading to fungistatic or fungicidal effects [23]. The azole agents demonstrate activity against most Candida species. The IDSA and ESCMID guidelines recommend fluconazole in patients who are not critically ill and are considered unlikely to be infected with an azole-resistant species. Fluconazole can also be used for step-down therapy in clinically stable patients. Voriconazole can be used as step-down treatment of $C$. glabrata or $C$. krusei infections (if susceptibility results suggest that they are active) or if additional mould coverage is required [2,3]. In the elderly, colonisation and infections with non-albicans species, including C. glabrata, has been shown to be increased [6, $33,34]$. In recent studies, the susceptibility to fluconazole and voriconazole of non-albicans species isolated from the elderly was approximately doubled when compared with the susceptibility of isolates obtained from younger patients $[6$, 7, 35].

Both voriconazole and fluconazole inhibit CYP3A4 and display many drug-drug interactions, resulting in a potential increase of adverse events when compared with the echinocandins [36]. Taking this into account, together with the increase in non-albicans species with reduced susceptibility in elderly patients, fluconazole and voriconazole are not recommended for the first-line treatment of invasive candidiasis in the elderly, but may be relevant for infections with specific isolates or as step-down therapy $[2,3]$.

4.2.2.1 Voriconazole In clinical trials, voriconazole plasma concentrations were $80-90 \%$ higher in elderly patients (over 65 years) than in younger patients. However, the prescribing information of voriconazole states that, although a relationship between plasma concentrations and age was observed, the safety profile of voriconazole in young and elderly patients is similar and, therefore, no dose adjustment is necessary [37]. Several studies demonstrated a relationship between the voriconazole plasma concentration and adverse effects, such as elevated liver enzymes and neurotoxicity including visual disturbances [38-40].

Voriconazole is metabolised by CYP (predominantly CYP2C19), which can be inhibited in patients with severe inflammation resulting in higher voriconazole concentrations [41]. Administration of voriconazole to patients with mild or moderate hepatic dysfunction resulted in a $223 \%$ increased exposure when compared with patients with normal liver function [37, 42, 43]. Co-morbidities and comedication in elderly may result in altered voriconazole concentrations [36]. TDM of voriconazole is recommended in elderly patients to ensure adequate exposure and avoid adverse events [2].

4.2.2.2 Fluconazole In a study in elderly patients (over 65 years), a higher exposure and longer terminal half-life of fluconazole was found than in young volunteers and the percentage of fluconazole recovered in urine and the renal clearance were generally lower in older patients [44]. Fluconazole is primarily eliminated by renal clearance, with $80 \%$ of the drug being excreted unchanged, with fluconazole clearance proportional to creatinine clearance. In adult patients, the plasma elimination half-life for fluconazole is approximately $30 \mathrm{~h}$. In patients with severe renal insuffi- 
ciency (glomerular filtration rate $<20 \mathrm{~mL} / \mathrm{min}$ ) the half-life increased to $98 \mathrm{~h}$ [44]. Consequently, a dose reduction of $50 \%$ is needed in patients with impaired renal function and patients on some renal replacement therapies [36, 44, 45]. TDM of fluconazole is currently not recommended in the guidelines; however, monitoring of fluconazole concentrations may be appropriate in select patients, such as the critically ill elderly and patients with renal insufficiency or on dialysis [46].

\subsubsection{Amphotericin B}

Amphotericin B is a polyene isolated from Streptomyces nodosus. Amphotericin B has a high affinity for ergosterol in the fungal membranes, leading to the formation of transmembrane pores, ion leakage and ultimately cell death [47]. Amphotericin B is recommended by the IDSA guideline in case of intolerance, limited availability or resistance to the antifungal agents [2].

Although not investigated thoroughly, the pharmacokinetic parameters for amphotericin B do not appear to change in elderly and age does not appear to be a relevant covariate in pharmacokinetic models for amphotericin B [48]. No dose adjustment is recommended with increasing age. Amphotericin B is not metabolised, and thus changes in drug metabolism or pharmacokinetic drug-drug interactions do not play a significant role [49]. The effectiveness of amphotericin B could be reduced in elderly as a recent study found a trend towards higher minimum inhibitory concentration (MIC) values for $C$. albicans in elderly than in younger patients treated for Candida infections, potentially due to the fact that older patients have been exposed more often to antibiotic and antifungal treatments, resulting in the selection of less susceptible strains [1]. Isolates were, however, still susceptible according to the EUCAST (European Committee on Antimicrobial Susceptibility Testing) clinical breakpoints (http://www.eucast.org/clinical_breakpoints/).

The use of amphotericin B deoxycholate is limited by its toxicity, including infusion-related reactions, hepatotoxicity, haematological effects and nephrotoxicity [47]. Nephrotoxicity during treatment with amphotericin B deoxycholate is observed especially in older adults, resulting in increased creatinine levels, hypokalaemia and/or hypomagnesaemia from the second week of therapy [50]. Electrolyte disturbances may be exacerbated by co-administration of thiazides and loop diuretics. Toxicity appears to be reduced for the lipid formulations (liposomal amphotericin B and amphotericin B lipid complex) [51-53]. These formulations do not appear to be more toxic in elderly patients than in younger patients and efficacy appears to be similar $[54,55]$. As observed for younger patients, concurrent use of nephrotoxic treatments should be avoided when possible, which is considered more relevant to elderly as these patients more often receive nephrotoxic treatments $[54,55]$.

\subsubsection{Flucytosine}

Flucytosine is a pyrimidine analogue that is taken up by cytosine permease and converted inside the fungal cell to its active moiety 5-fluoruracil, leading to inhibition of both DNA and RNA synthesis. Flucytosine may be added to the treatment for specific patient groups, including those with central nervous system (CNS) candidiasis and urinary tract infections [2]. Flucytosine is eliminated unchanged via glomerular filtration and therefore dosing should be adjusted accordingly in renal impairment. In patients with renal failure, the half-life may be increased up to 20 -fold. Since no metabolism occurs, pharmacokinetic drug-drug interactions are not expected. TDM is recommended for flucytosine as the pharmacokinetics are non-linear and the therapeutic window is narrow [56].

Candida species rapidly develop drug resistance to flucytosine administrated as monotherapy. Therefore, treatment with flucytosine should always be combined with another antifungal agent such as amphotericin B. Although concurrent use may lead to additive or synergistic effects on invasive mycoses, amphotericin B-induced nephrotoxicity may lead to the accumulation of flucytosine and increased toxicity, including myelosuppression and liver toxicity. Combined treatment with both drugs should therefore be limited to specific cases, such as cryptococcosis. When treatment with both drugs is necessary, TDM for flucytosine should be performed within $72 \mathrm{~h}$ after initiation and regularly thereafter [56].

\subsection{Therapeutic Drug Monitoring}

For anti-infective drugs, including antifungal agents, it is both difficult and time-consuming to assess directly whether the infection is adequately treated [57]. TDM can be performed to guide dosing of antifungal drugs $[58,59]$. For TDM to be of benefit, several criteria should be fulfilled (Fig. 2) [57-59]. Of the agents used to treat invasive candidiasis, voriconazole, posaconazole and flucytosine meet these criteria [60]. During treatment, it is recommended to repeat measurements of blood concentrations regularly, including after the start of treatment, dose adjustments, changed gastrointestinal absorption, changed interacting co-medication and changed clinical condition of the patient [56, 59]. For drugs with a wide therapeutic range, such as fluconazole, it seems more practical to empirically administer higher doses than performing TDM [59]. A therapeutic range, however, is described for this drug (area under the concentration-time curve $[\mathrm{AUC}] / \mathrm{MIC}>100 ; 400 \mathrm{AUC} \mathrm{mg} \cdot \mathrm{h} / \mathrm{L}$; 
Fig. 2 Criteria a drug should fulfil to perform therapeutic drug monitoring (TDM). TDM can be performed during the treatment of an invasive fungal infection or in the prophylaxis setting. For several antifungal agents TDM is recommended during treatment (see text). TDM should be repeated regularly during treatment. TDM can be considered in a prophylaxis setting

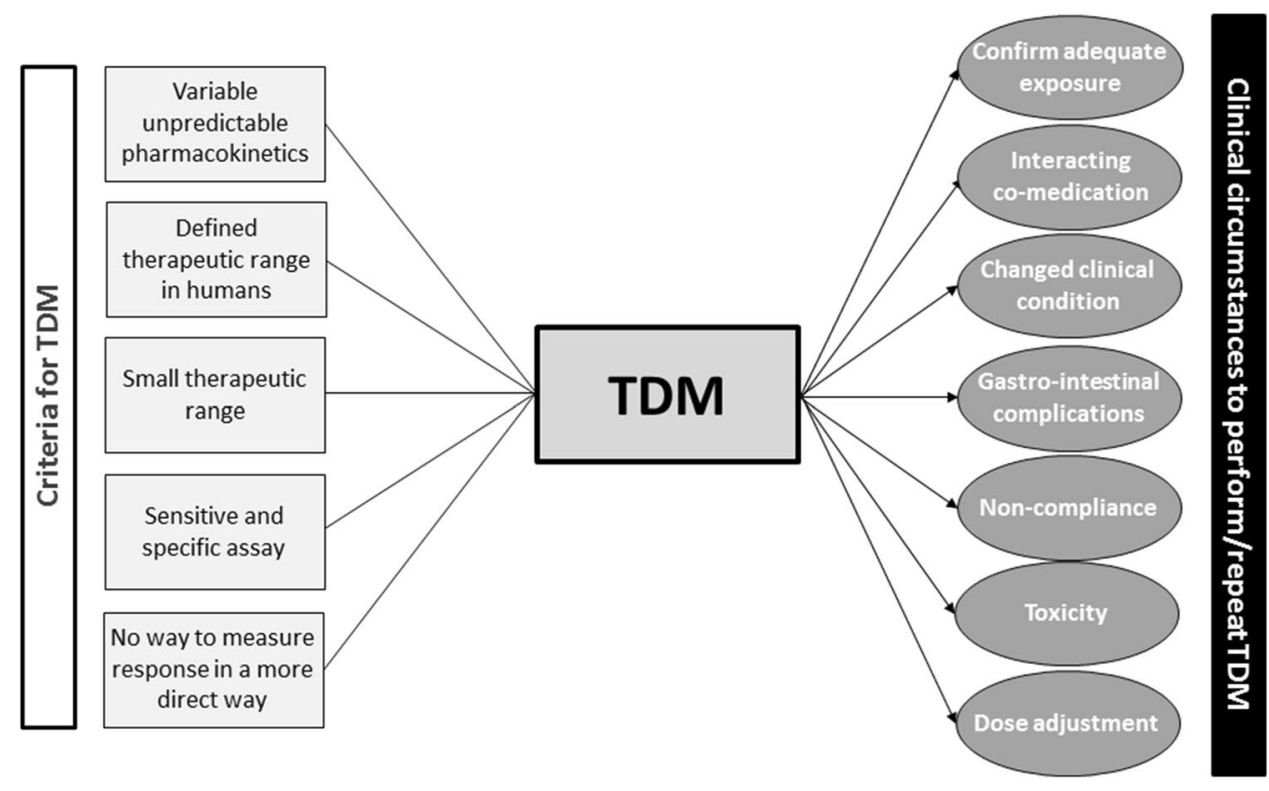

trough $>10-15 \mathrm{mg} / \mathrm{L})$ [59]. A therapeutic range for voriconazole (trough 1-5 $\mathrm{mg} / \mathrm{L}$, both prophylaxis and treatment) and posaconazole is defined for prophylaxis and treatment (trough concentration $>0.7$ and $>1.25 \mathrm{mg} / \mathrm{L}$, respectively) [56, 59, 61, 62]. Figure 2 illustrates when TDM should be considered.

TDM is not recommended for the echinocandins or amphotericin B by the current guidelines, although studies report significant variability in echinocandin exposure in specific patient populations [56, 63-65]. Decreased and potentially inadequate echinocandin exposure induced by physiological changes in critically ill patients may result in ineffective treatment and drug resistance [63-65]. Although in vitro studies have shown a concentration-effect relationship, a clear relationship between exposure and therapeutic response is currently lacking [60]. However, sensitivity of Candida isolates to echinocandins is decreasing, requiring higher doses and indicating that defined therapeutic ranges are required to perform TDM [66]. Analytical methods for the determination of plasma concentrations of echinocandins are available and can be implemented directly if TDM appears to be advantageous $[67,68]$. For now, TDM could be considered for the echinocandins or amphotericin B if patients do not respond to treatment, based on the effective pharmacokinetic/pharmacodynamic (PK/PD) parameters from in vivo studies [69]. Importantly, to appropriately interpret TDM, the susceptibility of the pathogen to the antifungal drug should be known as well.

In general, TDM is performed by using venous blood samples. In addition, dried blood spot sampling can be performed for voriconazole and posaconazole TDM. Voriconazole saliva concentrations have also been examined as an alternative, easily collected specimen [70, 71]. When implemented in clinical practice, these alternative sampling methods may be a more acceptable and less invasive sampling method for outpatient monitoring of older patients [57].

\section{Antimicrobial Stewardship}

Antimicrobial stewardship (AMS) is an effective and systemic approach to optimise the use of antimicrobial agents [72]. AMS programmes have been demonstrated to improve patient outcomes, reduce inappropriate antimicrobial use and minimise the adverse consequences, such as antimicrobial resistance, adverse drug effects and unnecessary costs.

\subsection{Antifungal Stewardship in Older People}

The aims of AFS overlap with those of AMS; however, there are nuanced differences, including that AFS generally involves fewer specialties, involves antifungal agents that are generally of high cost, and the antifungal pharmacokinetics are complex. Generally, there is a paucity of literature evaluating effective AFS recommendations in the elderly; however, the same general principles are expected to apply. The lack of new agents and growing resistance highlights the need to use current antifungal agents judiciously. The goal of all AFS programmes should be coordinated quality care and better outcomes for patients. Key target areas for AFS programs in the elderly include adjusting therapy based on diagnostic results, selecting an appropriate agent and dose for an appropriate duration, switching from intravenous to oral administration when indicated, initiation of TDM and guideline concordance. A secondary goal may be reduced 
antifungal resistance and cost containment. These goals are usually achieved by a multidisciplinary team, which should include a clinical pharmacist, with knowledge of antifungal $\mathrm{PK} / \mathrm{PD}$ and potential drug-drug interactions, and a medical specialist with expertise in interpreting antifungal diagnostic testing and clinical mycology. This AFS team should monitor and evaluate antifungal prescribing in relation to local resistance patterns, providing regular feedback to prescribers and monitor patient safety incidents. This should at least be done with key stakeholders, such as haematologists, intensivists, gastrointestinal tract surgeons and respiratory physicians, as appropriate.

\subsection{Considerations}

Empiric antifungal therapy should be driven by guidelines and incidence of antifungal treatment resistance. One study reported that patients who receive appropriate therapy have a significantly higher overall survival at 12 weeks [73]. Especially with the use of azoles, drug-drug interactions are a significant consideration, which should be reviewed and managed. An example of a recommendation could include withholding a statin (HMG-CoA reductase inhibitors) while the patient completes azole therapy. Key considerations for $\mathrm{PK} / \mathrm{PD}$ include monitoring of liver function when using caspofungin. In this case, anidulafungin could be substituted. Renal function needs to be considered for intravenous voriconazole and conventional amphotericin; however, these can be overcome by switching patients to oral voriconazole or using an alternative formulation of amphotericin (such as liposomal), respectively. TDM also plays an important role in optimising antifungal use. Finally, antifungal agents used in invasive candidiasis are generally of high cost and, despite being used less frequently than antibacterial agents, they tend to form a significant proportion of the pharmacy budgets. A number of published studies show that AFS programmes reduce annual hospital expenditure [74, 75]; however, this should not be the sole outcome measure of AFS success.

\subsection{Recommendations}

The aims of AFS programmes should be to optimise antifungal use, improve patient care, de-escalate and stop antifungal therapy when appropriate, and ensure TDM is performed when indicated. This can be achieved by multidisciplinary AFS teams, who conduct prospective audits and give feedback to prescribers within hospitals. The AFS team undertakes post-prescription review of patients receiving antifungal agents and should assess for appropriateness of the drug, dose and planned duration. Recommendations include stopping unnecessary empiric therapy, de-escalation based on guidelines or diagnostic tests, switching from intravenous to oral agents and optimising drug use. Other strategic, longer-term goals could include education of prescribers in the facility, regular feedback on antifungal use benchmarked to other peer groups or national data, implementation of evidence-based guidelines and review of local fungal resistance rates.

\section{Summary and Conclusion}

Candida infections are a growing problem in elderly patients, with significantly higher mortality in this group. Improved diagnostic tests are required to rapidly identify the Candida species. When treatment is initiated in the elderly, careful monitoring of benefit and harm should be an important consideration in the selection of both the drug and dose regimen. For selected antifungal agents, TDM is required to optimise treatment and prevent adverse effects. An AFS should be installed to optimise antifungal treatment.

\section{Compliance with Ethical Standards}

Funding BG.J. Dekkers, A. Veringa, D.J.E. Marriott, J.M. Boonstra, K.C.M. van der Elst, F.F. Doukas, A.J. McLachlan and J.W.C. Alffenaar received no external funding for this study.

Conflict of interest B.G.J. Dekkers, A. Veringa, D.J.E. Marriott, J.M. Boonstra, K.C.M. van der Elst, F.F. Doukas, A.J. McLachlan and J.W.C. Alffenaar declare no conflicts of interest relevant to this paper.

Open Access This article is distributed under the terms of the Creative Commons Attribution-NonCommercial 4.0 International License (http://creativecommons.org/licenses/by-nc/4.0/), which permits any noncommercial use, distribution, and reproduction in any medium, provided you give appropriate credit to the original author(s) and the source, provide a link to the Creative Commons license, and indicate if changes were made.

\section{References}

1. Barchiesi F, Orsetti E, Mazzanti S, Trave F, Salvi A, Nitti C, et al. Candidemia in the elderly: what does it change? PLoS One. 2017; 12:e0176576.

2. Pappas PG, Kauffman CA, Andes DR, Clancy CJ, Marr KA, Ostrosky-Zeichner L, et al. Executive summary: clinical practice guideline for the management of candidiasis: 2016 update by the Infectious Diseases Society of America. Clin Infect Dis. 2016;62:409-17.

3. Cornely OA, Bassetti M, Calandra T, Garbino J, Kullberg BJ, Lortholary O, et al. ESCMID guideline for the diagnosis and management of Candida diseases 2012: non-neutropenic adult patients. Clin Microbiol Infect. 2012;18(Suppl 7):19-37.

4. Ramos-Martínez A, Vicente-López N, Sánchez-Romero I, Padilla B, Merino-Amador P, Garnacho-Montero J, et al. Epidemiology 
and prognosis of candidaemia in elderly patients. Mycoses. 2017;60:808-17.

5. Lerma A, Cantero E, Soriano M, Orden B, Muñez E, RamosMartinez A. Clinical presentation of candidaemia in elderly patients: experience in a single institution. Rev Esp Quimioter. 2017;30:207-12.

6. Wang H, Liu N, Yin M, Han H, Yue J, Zhang F, et al. The epidemiology, antifungal use and risk factors of death in elderly patients with candidemia: a multicentre retrospective study. BMC Infect Dis. 2014; 14:609.

7. Guimarães T, Nucci M, Mendonça JS, Martinez R, Brito LR, Silva $\mathrm{N}$, et al. Epidemiology and predictors of a poor outcome in elderly patients with candidemia. Int J Infect Dis. 2012;16:e442-7.

8. Mylonakis E, Clancy CJ, Ostrosky-Zeichner L, Garey KW, Alangaden GJ, Vazquez JA, et al. T2 magnetic resonance assay for the rapid diagnosis of candidemia in whole blood: a clinical trial. Clin Infect Dis. 2015;60:892-9.

9. Karageorgopoulos DE, Vouloumanou EK, Ntziora F, Michalopoulos A, Rafailidis PI, Falagas ME. $\beta$-D-glucan assay for the diagnosis of invasive fungal infections: a meta-analysis. Clin Infect Dis. 2011;52:750-70.

10. Nguyen MH, Wissel MC, Shields RK, Salomoni MA, Hao B, Press EG, et al. Performance of Candida real-time polymerase chain reaction, $\beta$-D-glucan assay, and blood cultures in the diagnosis of invasive candidiasis. Clin Infect Dis. 2012;54:1240-8.

11. McLachlan AJ, Hilmer SN, Le Couteur DG. Variability in response to medicines in older people: phenotypic and genotypic factors. Clin Pharmacol Ther. 2009;85:431-3.

12. McLachlan AJ, Pont LG. Drug metabolism in older people-a key consideration in achieving optimal outcomes with medicines. J Gerontol A Biol Sci Med Sci. 2012;67:175-80.

13. Hilmer SN, McLachlan AJ, Le Couteur DG. Clinical pharmacology in the geriatric patient. Fundam Clin Pharmacol. 2007;21:217-30.

14. McLean AJ, Le Couteur DG. Aging biology and geriatric clinical pharmacology. Pharmacol Rev. 2004;56:163-84.

15. Hilmer S, Gnjidic D. The effects of polypharmacy in older adults. Clin Pharmacol Ther. 2009;85:86-8.

16. Gnjidic D, Hilmer SN, Blyth FM, Naganathan V, Waite L, Seibel MJ, et al. Polypharmacy cutoff and outcomes: five or more medicines were used to identify community-dwelling older men at risk of different adverse outcomes. J Clin Epidemiol. 2012;65:989-95.

17. Hubbard RE, O'Mahony MS, Woodhouse KW. Medication prescribing in frail older people. Eur J Clin Pharmacol. 2013;69:319-26.

18. Johnston C, Hilmer SN, McLachlan AJ, Matthews ST, Carroll PR, Kirkpatrick CM. The impact of frailty on pharmacokinetics in older people: using gentamicin population pharmacokinetic modeling to investigate changes in renal drug clearance by glomerular filtration. Eur J Clin Pharmacol. 2014;70:549-55.

19. Roberts JA, Abdul-Aziz MH, Lipman J, Mouton JW, Vinks AA, Felton TW, et al. Individualised antibiotic dosing for patients who are critically ill: challenges and potential solutions. Lancet Infect Dis. 2014;14:498-509.

20. Butler JM, Begg EJ. Free drug metabolic clearance in elderly people. Clin Pharmacokinet. 2008;47:297-321.

21. Aw D, Silva AB, Palmer DB. Immunosenescence: emerging challenges for an ageing population. Immunology. 2007;120:435-46.

22. Stott KE, Hope WW. Therapeutic drug monitoring for invasive mould infections and disease: pharmacokinetic and pharmacodynamic considerations. J Antimicrob Chemother. 2017;72:i12-8.

23. Ashley ESD, Lewis R, Lewis JS, Martin C, Andes D. Pharmacology of systemic antifungal agents. Clin Infect Dis. 2006;43:S28-39.

24. Mourad A, Perfect JR. Tolerability profile of the current antifungal armoury. J Antimicrob Chemother. 2018;73:i26-32.
25. Sucher AJ, Chahine EB, Balcer HE. Echinocandins: the newest class of antifungals. Ann Pharmacother. 2009;43:1647-57.

26. Castanheira M, Messer SA, Rhomberg PR, Pfaller MA. Antifungal susceptibility patterns of a global collection of fungal isolates: results of the SENTRY Antifungal Surveillance Program (2013). Diagn Microbiol Infect Dis. 2016;85:200-4.

27. Wang J-L, Chang C-H, Young-Xu Y, Chan KA. Systematic review and meta-analysis of the tolerability and hepatotoxicity of antifungals in empirical and definitive therapy for invasive fungal infection. Antimicrob Agents Chemother. 2010;54:2409-19.

28. Chen SC-A, Slavin MA, Sorrell TC. Echinocandin antifungal drugs in fungal infections: a comparison. Drugs. 2011;71:11-41.

29. DiNubile MJ, Strohmaier KM, Lupinacci RJ, Meibohm AR, Sable CA, Kartsonis NA. Efficacy and safety of caspofungin therapy in elderly patients with proven or suspected invasive fungal infections. Eur J Clin Microbiol Infect Dis. 2008;27:663-70.

30. Dimopoulos G, Paiva J-A, Meersseman W, Pachl J, Grigoras I, Sganga G, et al. Efficacy and safety of anidulafungin in elderly, critically ill patients with invasive Candida infections: a post hoc analysis. Int J Antimicrob Agents. 2012;40:521-6.

31. Lee C-H, Lin J-C, Ho C-L, Sun M, Yen W-T, Lin C. Efficacy and safety of micafungin versus extensive azoles in the prevention and treatment of invasive fungal infections for neutropenia patients with hematological malignancies: a meta-analysis of randomized controlled trials. PLoS One. 2017;12:e0180050.

32. Lepak AJ, Zhao M, VanScoy B, Ambrose PG, Andes DR. Pharmacodynamics of a long-acting echinocandin, CD101, in a neutropenic invasive-candidiasis murine model using an extended-interval dosing design. Antimicrob Agents Chemother. 2018;62:e02154-17.

33. Diekema DJ, Messer SA, Brueggemann AB, Coffman SL, Doern GV, Herwaldt LA, et al. Epidemiology of candidemia: 3-year results from the emerging infections and the epidemiology of Iowa organisms study. J Clin Microbiol. 2002;40:1298-302.

34. Benito-Cruz B, Aranda-Romo S, López-Esqueda FJ, de la RosaGarcía E, Rosas-Hernández R, Sánchez-Vargas LO. Oral Candida isolates and fluconazole susceptibility patterns in older Mexican women. Arch Gerontol Geriatr. 2016;65:204-10.

35. Luzzati R, Cavinato S, Deiana ML, Rosin C, Maurel C, Borelli M. Epidemiology and outcome of nosocomial candidemia in elderly patients admitted prevalently in medical wards. Aging Clin Exp Res. 2015;27:131-7.

36. Brüggemann RJM, Alffenaar J-WC, Blijlevens NMA, Billaud EM, Kosterink JGW, Verweij PE, et al. Clinical relevance of the pharmacokinetic interactions of azole antifungal drugs with other coadministered agents. Clin Infect Dis. 2009;48:1441-58.

37. Pfizer. Summary of product characteristics: Vfend. New York: Pfizer; 2008.

38. Park WB, Kim N-H, Kim K-H, Lee SH, Nam W-S, Yoon SH, et al. The effect of therapeutic drug monitoring on safety and efficacy of voriconazole in invasive fungal infections: a randomized controlled trial. Clin Infect Dis. 2012;55:1080-7.

39. Pascual A, Calandra T, Bolay S, Buclin T, Bille J, Marchetti O. Voriconazole therapeutic drug monitoring in patients with invasive mycoses improves efficacy and safety outcomes. Clin Infect Dis. 2008;46:201-11.

40. Dolton MJ, Ray JE, Chen SC-A, Ng K, Pont LG, McLachlan AJ. Multicenter study of voriconazole pharmacokinetics and therapeutic drug monitoring. Antimicrob Agents Chemother. 2012;56:4793-9.

41. Veringa A, ter Avest M, Span LFR, van den Heuvel ER, Touw DJ, Zijlstra JG, et al. Voriconazole metabolism is influenced by severe inflammation: a prospective study. J Antimicrob Chemother. 2017;72:261-7. 
42. Jeu L, Piacenti FJ, Lyakhovetskiy AG, Fung HB. Voriconazole. Clin Ther. 2003;25:1321-81.

43. Theuretzbacher U, Ihle F, Derendorf H. Pharmacokinetic/pharmacodynamic profile of voriconazole. Clin Pharmacokinet. 2006;45:649-63.

44. Pfizer. Summary of product characteristics: Diflucan. Kent: Pfizer; 2008.

45. Trotman RL, Williamson JC, Shoemaker DM, Salzer WL. Antibiotic dosing in critically ill adult patients receiving continuous renal replacement therapy. Clin Infect Dis. 2005;41:1159-66.

46. Andes D, Pascual A, Marchetti O. Antifungal therapeutic drug monitoring: established and emerging indications. Antimicrob Agents Chemother. 2009;53:24-34.

47. Loo AS, Muhsin SA, Walsh TJ. Toxicokinetic and mechanistic basis for the safety and tolerability of liposomal amphotericin B. Expert Opin Drug Saf. 2013;12:881-95.

48. Würthwein G, Young C, Lanvers-Kaminsky C, Hempel G, Trame MN, Schwerdtfeger R, et al. Population pharmacokinetics of liposomal amphotericin B and caspofungin in allogeneic hematopoietic stem cell recipients. Antimicrob Agents Chemother. 2012;56:536-43.

49. Bellmann R. Clinical pharmacokinetics of systemically administered antimycotics. Curr Clin Pharmacol. 2007;2:37-58.

50. Kauffman CA. Fungal infections in older adults. Clin Infect Dis. 2001;33:550-5.

51. White MH, Bowden RA, Sandler ES, Graham ML, Noskin GA, Wingard JR, et al. Randomized, double-blind clinical trial of amphotericin B colloidal dispersion vs. amphotericin B in the empirical treatment of fever and neutropenia. Clin Infect Dis. 1998;27:296-302.

52. Walsh TJ, Finberg RW, Arndt C, Hiemenz J, Schwartz C, Bodensteiner D, et al. Liposomal amphotericin B for empirical therapy in patients with persistent fever and neutropenia. N Engl J Med. 1999;340:764-71.

53. Sharkey PK, Graybill JR, Johnson ES, Hausrath SG, Pollard RB, Kolokathis A, et al. Amphotericin B lipid complex compared with amphotericin B in the treatment of cryptococcal meningitis in patients with AIDS. Clin Infect Dis. 1996;22:315-21.

54. Hooshmand-Rad R, Chu A, Gotz V, Morris J, Batty S, Freifeld A. Use of amphotericin B lipid complex in elderly patients. J Infect. 2005;50:277-87.

55. Ueda S, Miyamoto S, Kaida K, Chizuka A, Kojima R, Takano J, et al. Safety and efficacy of treatment with liposomal amphotericin $B$ in elderly patients at least 65 years old with hematological diseases. J Infect Chemother. 2016;22:287-91.

56. Ashbee HR, Barnes RA, Johnson EM, Richardson MD, Gorton R, Hope WW. Therapeutic drug monitoring (TDM) of antifungal agents: guidelines from the British Society for Medical Mycology. J Antimicrob Chemother. 2013;69:1162-76.

57. Veringa A, Sturkenboom MGG, Dekkers BGJ, Koster RA, Roberts JA, Peloquin CA, et al. LC-MS/MS for therapeutic drug monitoring of anti-infective drugs. Trends Anal Chem. 2016;84:34-40.

58. Brüggemann RJM, Aarnoutse RE. Fundament and prerequisites for the application of an antifungal TDM service. Curr Fungal Infect Rep. 2015;9:122-9.

59. Lewis R, Bruggemann RJ, Padoin C, Maertens J, Marchetti O, Groll AH, et al. ECIL 6: triazole antifungal therapeutic drug monitoring. 2015. https://www.ebmt.org/Contents/Resources/Library/ ECIL/Pages/ECIL.aspx. Accessed 1 Mar 2018.
60. Drew RH, Townsend ML, Pound MW, Johnson SW, Perfect JR. Recent advances in the treatment of life-threatening, invasive fungal infections. Expert Opin Pharmacother. 2013;14:2361-74.

61. Troke PF, Hockey HP, Hope WW. Observational study of the clinical efficacy of voriconazole and its relationship to plasma concentrations in patients. Antimicrob Agents Chemother. 2011;55:4782-8.

62. Dekkers BGJ, Bakker M, van der Elst KCM, Sturkenboom MGG, Veringa A, Span LFR, et al. Therapeutic drug monitoring of posaconazole: an update. Curr Fungal Infect Rep. 2016;10:51-61.

63. van der Elst KCM, Veringa A, Zijlstra JG, Beishuizen A, Klont $\mathrm{R}$, Brummelhuis-Visser $\mathrm{P}$, et al. Low caspofungin exposure in patients in the intensive care unit. Antimicrob Agents Chemother. 2016;61:e01582-16.

64. van Wanrooy MJP, Rodgers MGG, Uges DRA, Arends JP, Zijlstra JG, van der Werf TS, et al. Low but sufficient anidulafungin exposure in critically ill patients. Antimicrob Agents Chemother. 2014;58:304-8.

65. Boonstra JM, van der Elst KC, Veringa A, Jongedijk EM, Brüggemann RJ, Koster RA, et al. Pharmacokinetic properties of micafungin in critically ill patients diagnosed with invasive candidiasis. Antimicrob Agents Chemother. 2017;61:e01398-17.

66. Perlin DS, Rautemaa-Richardson R, Alastruey-Izquierdo A. The global problem of antifungal resistance: prevalence, mechanisms, and management. Lancet Infect Dis. 2017;17:e383-92.

67. van Wanrooy MJP, Santoe RN, van der Elst KCM, Wilmer CM, van Hateren K, Wessels AMA, et al. Simultaneous quantification of anidulafungin and caspofungin in plasma by an accurate and simple liquid chromatography tandem mass-spectrometric method. Ther Drug Monit. 2013;35:778-84.

68. Boonstra JM, Jongedijk EM, Koster RA, Touw DJ, Alffenaar JWC. Simple and robust LC-MS/MS method for therapeutic drug monitoring of micafungin. Bioanalysis. 2018;10:877-86.

69. Pea F, Lewis RE. Overview of antifungal dosing in invasive candidiasis. J Antimicrob Chemother. 2018;73:i33-43.

70. Vanstraelen K, Maertens J, Augustijns P, Lagrou K, de Loor $\mathrm{H}$, Mols $\mathrm{R}$, et al. Investigation of saliva as an alternative to plasma monitoring of voriconazole. Clin Pharmacokinet. 2015;54:1151-60.

71. van der Elst KCM, Span LFR, van Hateren K, Vermeulen KM, van der Werf TS, Greijdanus B, et al. Dried blood spot analysis suitable for therapeutic drug monitoring of voriconazole, fluconazole, and posaconazole. Antimicrob Agents Chemother. 2013;57:4999-5004.

72. Duguid M, Cruickshank M, editors. Antimicrobial stewardship in Australian hospitals. Sydney: Australian Commission on Safety and Quality in Healthcare; 2010.

73. Nivoix Y, Launoy A, Lutun P, Moulin J-C, Phai Pang K-A, Fornecker L-M, et al. Adherence to recommendations for the use of antifungal agents in a tertiary care hospital. J Antimicrob Chemother. 2012;67:2506-13.

74. Micallef C, Aliyu SH, Santos R, Brown NM, Rosembert D, Enoch DA. Introduction of an antifungal stewardship programme targeting high-cost antifungals at a tertiary hospital in Cambridge. England. J Antimicrob Chemother. 2015;70:1908-11.

75. Standiford HC, Chan S, Tripoli M, Weekes E, Forrest GN. Antimicrobial stewardship at a large tertiary care academic medical center: cost analysis before, during, and after a 7-year program. Infect Control Hosp Epidemiol. 2012;33:338-45. 